\title{
Variáveis clínicas e razões para busca de tratamento de pacientes tabagistas
}

Camila Barbosa dos Santos ${ }^{1}$ Silvana Alba Scortegagna ${ }^{1}$

Renata da Rocha Campos Franco ${ }^{2}$ Lia Mara Wibelinger ${ }^{1}$

Universidade de Passo Fundo, Passo Fundo, RS, Brasil.

${ }^{2}$ Institut Catholique de Toulouse, Toulouse, França.
Objetivo: identificar variáveis clínicas e razões para
busca de tratamento de tabagistas. Métodos: estudo
retrospectivo documental, com pacientes assistidos no
Centro de Atenção Psicossocial Álcool e outras drogas
de 2013 a 2016 . Resultados: dos 211 usuários, 41
apresentaram dependência à nicotina: mulheres (68,3\%),
baixa escolaridade ( $70,7 \%)$, início precoce do tabagismo
(82,9\%), elevada/muito elevada ( $61 \%)$, presença de
problemas psíquicos ( $78 \%$ ) e ansiedade (58,5\%). Na
busca de tratamento, $85 \%$ das mulheres demonstraram
preocupação com saúde e $73 \%$ custos elevados do cigarro.

Conclusão: políticas públicas de intervenção e prevenção devem ser ampliadas e desenvolvidas com atenção especial às mulheres.

Descritores: Dependência de Usuários; Tabagismo; Motivação; Intervenção.

\section{Como citar este artigo}

Santos CB, Scortegagna SA, Franco RRC, Wibelinger LM. Clinical variables and reasons smokers seek treatment. SMAD, Rev Eletrônica Saúde Mental Álcool Drog. 2019;15(2):77-86. doi: https://dx.doi.org/10.11606/issn.1806-6976.smad.2019.149180 


\section{Clinical variables and reasons smokers seek treatment}

Objective: to identify clinical variables and reasons for seeking treatment of smokers. Methods: retrospective documentary study, with patients assisted at the Center for Psychosocial Care Alcohol and other drugs from 2013 to 2016. Results: of the 211 users, 41 had nicotine dependence: women (68.3\%), low level of schooling (70.7\%). Early onset of smoking (82.9\%), high / very high (61\%), presence of psychic problems (78\%) and anxiety $(58,5 \%)$. In the search for treatment, $85 \%$ of the women showed health concern and $73 \%$ high cigarette costs. Conclusion: public intervention and prevention policies should be expanded and developed with special attention to women.

Descriptors: Dependence of Users; Smoking; Motivation; Intervention.

\section{Variables clínicos y razones para búsqueda de tratamiento de pacientes tabagistas}

Objetivo: identificar variables clínicas y razones para la búsqueda de tratamiento de los fumadores. Métodos: estudio retrospectivo documental, con pacientes asistidos en el Centro de Atención Psicosocial Alcohol y otras drogas de 2013 a 2016. Resultados: en los 211 usuarios, 41 presentaron adicción a la nicotina: mujeres (68,3\%), baja escolaridad $(70,7 \%)$. El inicio temprano del tabaquismo (82,9\%), elevada / muy elevada (61\%), presencia de problemas psíquicos $(78 \%)$ y ansiedad (58,5\%). En la búsqueda de tratamiento, el $85 \%$ de las mujeres mostraron preocupación por la salud y el 73\% de los altos costos del cigarrillo. Conclusión: las políticas públicas de intervención y prevención deben ser ampliadas y desarrolladas con atención especial a las mujeres.

Descriptores: Dependencia de Usuários; Tabaquismo; Motivación; Intervención. 


\section{Introdução}

O tabagismo é uma doença crônica, um problema de saúde pública, tanto nacional quanto internacionalmente, em razão da elevada prevalência e da alta mortalidade decorrente das doenças relacionadas ao tabaco. Estima-se que exista 1,3 bilhão de fumantes no mundo com 15 ou mais anos de idade, constituindo um terço da população global. Desses, 900 milhões estão em países em desenvolvimento e 250 milhões são mulheres. O consumo anual é de 7 trilhões e 30 bilhões de cigarros, correspondendo a 20 bilhões por dia; cerca de 75.000 toneladas de nicotina são consumidas por ano, das quais 200 toneladas são diárias ${ }^{(1-2)}$.

Comparados aos não fumantes, os fumantes têm menos qualidade de vida, maior prevalência de sofrimento psicológico e aumento da mortalidade(3). A expectativa de vida de indivíduos que fumam é $25 \%$ menor que a de não fumantes, que também vivem mais anos sem problemas de saúde ${ }^{(4)}$. No Brasil ocorrem cerca de 6 milhões de mortes anuais provocadas pelo tabagismo, podendo chegar a 7,5 milhões por ano, em $2020^{(1)}$. A dependência ao fumo responde por $71 \%$ dos casos de câncer de pulmão, $42 \%$ das doenças respiratórias crônicas, $10 \%$ das doenças cardiovasculares e por cerca de $10 \%$ de todas as mortes. Além disso, está entre as principais causas de morte antecipada entre os idosos, exigindo cuidados médicos e de saúde.

O tabagismo associa-se a enormes custos sociais e econômicos originários do aumento da morbidade e mortalidade relacionadas ao fumo, incluindo custos gerados pelos fumantes, como uso de recursos de saúde, ausência no trabalho, perda da produtividade, pagamento de auxílio-doença entre outros ${ }^{(5)}$. Assim sendo, o consumo excessivo do tabaco traz problemas de saúde física e psíquica, financeiros e de relacionamento. Mas os dependentes nem sempre estão conscientes destes problemas e quando percebem os efeitos nocivos, já caíram no círculo vicioso(6).

Embora esteja em declínio em países como Austrália, Estados Unidos, Inglaterra, Nova Zelândia e Canadá, a prevalência de tabagismo em mulheres adultas ainda é maior nos países desenvolvidos, com taxas médias de $22 \%$. De maneira geral, entre mulheres jovens, a prevalência cresce mundialmente e uma das razões pelo que se deve considerar como importante contexto para se entender a influência do tabagismo nas relações entre grupos de pares é o ambiente escolar. Segundo a literatura, este ambiente é um importante espaço para implementações de ações eficazes no combate ao tabagismo, e, para tanto, deve-se conhecer as motivações, as crenças e as influências associadas ao consumo de tabaco(7).

As comorbidades relacionadas ao tabagismo também devem ser consideradas no contexto do tratamento. A maior parte dos fumantes apresentam transtornos psiquiátricos e características particulares que exigem atenção diferenciada e criação de subgrupos específicos ${ }^{(3)}$. As comorbidades psiquiátricas, frequentemente, estão relacionadas à presença de transtornos de humor, de ansiedade, transtornos de uso de substâncias psicoativas e esquizofrenia e podem comprometer a eficácia da intervenção terapêutica(8-9). Um estudo transversal realizado no Distrito Federal relacionou o nível de ansiedade e de depressão com a motivação para deixar de fumar com uma amostra de 1.233 fumantes atendidos em vinte centros de referência para tratamento do tabagismo. A motivação foi elevada em $50 \%$ dos pacientes, a ansiedade provável em 34\% e a depressão em $22 \%{ }^{(9)}$.

Existem evidências que demonstram a alta prevalência de transtornos psiquiátricos entre a população que faz uso de substâncias psicoativas. Também é alta a prevalência de dependência de nicotina e álcool entre os dependentes de outras drogas ${ }^{(8)}$.

O Transtorno Mental Comum (TMC) é destacado com referência aos transtornos como enfermidade que acomete a população. Compreendido pela presença de diferentes sintomas por pelo menos sete dias, os sintomas principais destacados são: irritação, ansiedade, dificuldade de concentração, problemas em conciliar o sono, preocupação excessiva, sobretudo com a saúde, obsessões e compulsões, humor depressivo e fobia. A avaliação desses sintomas possibilita o diagnóstico precoce e o acompanhamento de transtornos depressivo, ansiedade, fobia, transtorno de pânico e transtorno obsessivo-compulsivo, caracterizados como alguns dos tipos de TMC(10).

A literatura(11) refere associação positiva entre tabagismo e doença mental, relacionando altas taxas de tabagismo com a gravidade da doença mental. Também descrevem que indivíduos com doença mental tendem a começar a fumar em idade mais adiantada, fumam mais pesadamente e são mais viciados em cigarros que a população em geral.

Existem várias hipóteses para explicar as altas taxas de tabagismo em pessoas com doenças mentais relacionadas à depressão e à ansiedade. A hipótese de automedicação postula que os indivíduos fumam para aliviar sintomas da vida cotidiana como: irritação, ansiedade, dificuldade de concentração, problemas em conciliar o sono, preocupação excessiva, sobretudo com a saúde, obsessões e compulsões, humor depressivo e fobia(11). Pesquisa relata que fumar pode desencadear depressão ou ansiedade, já que o efeito psicoativo do tabaco altera os neurocircuitos do indivíduo. Estudos com animais indicam que uma prolongada exposição à nicotina desregula o sistema hipotálamo-hipófiseadrenal, resultando em hipersecreção de cortisol e 
alterações na atividade do sistema neurotransmissor cuja função é regular as reações a estressores, um efeito que parece normalizar após a retirada de nicotina(11).

Estudos $^{(12)}$ sobre ansiedade e tabagismo mostram que os transtornos de ansiedade têm frequência elevada entre pacientes ambulatoriais (75\%), sendo os mais frequentes a fobia específica e o transtorno de ansiedade generalizada. Além disso, aproximadamente $1 / 4$ (um quarto) de pacientes tabagistas em uso de tabaco apresentam importante associação com transtorno de ansiedade generalizada (TAG), indicando a correlação desses transtornos com o uso de tabaco. Sobre o tabaco, outro dado significativo é o número de cigarros fumados e a presença ou não de transtornos de ansiedade, em que se evidencia pacientes com essas patologias com tendência a fumar mais cigarros, embora esse dado não tenha significância estatística.

Em uma pesquisa com 277 pacientes psiquiátricos, no Brasil, consultados na Clínica Ambulatorial do Instituto de Psiquiatria, Universidade Federal do Rio de Janeiro, com transtornos de ansiedade e depressão, foram encontrados tabagistas que consomem de forma abusiva(13). Por sua vez, um estudo(14) realizado nas unidades públicas do Distrito Federal descreve que fumantes e não fumantes não se diferenciaram quanto às variáveis sociodemográficas, demonstrando que o cigarro é um problema de saúde pública e que atinge a todas as classes sociais e culturais em diferentes idades e em ambos os sexos. Já a ansiedade, a depressão, o tempo e a quantidade de consumo de tabaco atingiram valores próximos de uma significância estatística. Do ponto de vista qualitativo, este estudo mostra que o consumo do tabaco está associado a uma tentativa de compensar a presença de um desconforto interno (ansiedade e depressão), assim como o tempo e quantidade de consumo está relacionado com a dependência.

Uma revisão sistemática de literatura de estudos longitudinais ${ }^{(11)}$, sobre as associações entre tabagismo e depressão/ansiedade revisou 6.232 resumos; destes, 5.514 foram excluídos com base no título e 404, após a leitura do resumo. No total, 314 artigos foram recuperados e avaliados para a elegibilidade e 148 preencheram os critérios de inclusão. O tamanho da amostra variou de 59 a 90.627 participantes, que seguiram em acompanhamento de 2 meses a 36 anos. Dos 148 estudos incluídos, 99 (67\%) recrutaram participantes masculinos e femininos, $16(11 \%)$ apenas mulheres e 7 ( $5 \%)$ somente homens. Além disso, em 101 estudos (70\%) os participantes eram parte da população em geral, 15 (10\%) eram parte de populações clínicas e 16 (10\%) de determinados grupos étnicos.

As mulheres buscam mais tratamento para a cessação do tabagismo, devido ao fato de apresentarem maior envolvimento com as questões de saúde(15), acreditam mais no fato de que o tabagismo pode causar câncer(16), e participam mais dos grupos de apoio contra a dependência(15).

A literatura(17) indica que apesar de as taxas relacionadas ao consumo de cigarro serem um elemento comum na decisão de ambos os sexos, a decisão de fumar das mulheres é também sensível a preços, além de outros fatores sociais e culturais. Além disso, esses foram apreendidos no fato de que mulheres que residem em países que fazem parte da Organização para Cooperação e Desenvolvimento Econômico (OCDE) apresentam, de forma significativa, maior prevalência no consumo de cigarros.

Por sua vez, outro estudo(18) descreve a predominância de homens entre a população tabagista em todas as faixas etárias, enquanto na população não tabagista, prevalecem as mulheres, exceto na faixa etária dos 14 aos 17 anos. Isso demonstra que os gastos com tabagismo estão fortemente ligados ao sexo masculino $(63,5 \%)$ e que o trabalho preventivo com as meninas adolescentes é indispensável.

A literatura(19) também indica uma relação entre uso de medicamento e terapias para cessar o hábito de fumar em 154 mulheres, com pelo menos 30 anos de idade e fumantes de mais de 10 cigarros/dia. A conformidade com a medicação de estudo foi avaliada utilizando o Sistema de Monitorização de Eventos de Medicamentos (MEMS) ao longo de 7 (sete) semanas de tratamento. As intervenções psicológicas foram realizadas em sessões semanais de 60 minutos. Os resultados de abstinência do final do tratamento (EOT), até 12 meses após o tratamento, revelaram uma interação significativa de medicação e terapia. A eficiência da terapia medicamentosa, apesar de ser apreciada por sua eficiência, tornou-se ainda mais eficaz quando associada às psicoterapias, demonstrando que mesmo depois de 12 meses do fim do tratamento proposto, a abstinência ainda prevalecia ${ }^{(19)}$

Portanto, o êxito do tratamento para cessar de fumar está atrelado a um acompanhamento bio-psicosocial(19). Por isso, há necessidade de abordar elementos do contexto clínico como: a gravidade da dependência de nicotina, a idade de início do consumo do tabaco, as comorbidades, o histórico familiar, a motivação para cessar, assim como os sentimentos destacados relacionados ao tabagismo, também, em outros

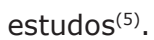

Ainda com referência aos fatores associados à abstinência do tabaco, na Alemanha, um estudo(20), realizado com 1.499 tabagistas, indicou que pessoas de maior escolaridade, ou seja, nível universitário e do sexo feminino apresentaram maior número de tentativas anteriores de parar de fumar, sem acompanhamento 
médico e aquelas que tinham a intenção de abandonar o fumo nos próximos seis meses apresentaram maiores chances de obter sucesso em seis meses quando acompanhadas por uma equipe de saúde.

A partir de estudo observacional(15) com pessoas que participaram do GATT no ano de 2009, em Vitória (ES), e avaliados quanto aos efeitos do tratamento, nove a vinte meses após término do mesmo, teve predomínio do sexo feminino em 115 (71,9\%) pacientes; faixa etária entre 45 e 64 anos com $101(63,1 \%)$ pacientes; média de idade 49,6 ( $\pm 10,9)$ anos; 81 (50,6\%) participantes com $2^{\circ}$ grau completo e incompleto; um aspecto importante a acrescentar é que as mulheres do Brasil, 79 (49,4\%) casadas apresentam mais dificuldade na cessação do tabagismo, quando comparada aos homens, além de serem mais suscetíveis à depressão e ansiedade, o que interfere na qualidade do engajamento terapêutico como, por exemplo, a terapia com reposição à nicotina (TRN).

A literatura(21) relata que tem crescido a preocupação com o consumo de tabaco pelas mulheres, fato que tem motivado uma maior atenção das instituições de saúde na tentativa de alertar a sociedade e os profissionais de saúde para o impacto negativo que o uso de tabaco causa sobre a saúde da mulher.

Outro estudo(16) não constatou diferenças significativas quanto ao número de tentativas para cessar o consumo de tabaco entre fumantes em função do uso de substâncias psicoativas ou de quadros de depressão, mas foi observada uma significativa associação do ponto clínico, com maior no número de hospitalizações no último mês e uma maior incidência de doenças agravadas pelo tabaco, como diabetes, hipertensão, doenças cardíacas e respiratórias.

Importante referir, como relatado na literatura, que a baixa procura dos homens por tratamentos para cessar o hábito do tabagismo é um dos desafios dos serviços de atenção básica de saúde. Essa e outras questões destacadas evidenciam a diferença de gênero na compreensão da cessação do tabagismo e a necessidade de estratégias para captação, tanto de homens como de mulheres, para realizar tratamentos a fim de abandonar o fumo tem ficado à margem dos serviços de saúde em geral $^{(15)}$.

Mas, independentemente do gênero e idade, uma vez consciente da sua dependência e dos efeitos nocivos do cigarro, o fumante irá se lançar no desafio de superar três tipos de dependência(6). A dependência física, em que o corpo vai ser dependente da nicotina presente no tabaco, é tamanha que sem esta substância, o corpo sofrerá os sintomas de abstinência, principalmente irritabilidade; nervosismo e ansiedade; agitação; distúrbios do sono; humor deprimido; perturbação de concentração intelectual; aumento do apetite ou constipação. Esses sintomas, vividos como desagradáveis, provocam na maioria das vezes as recaídas de curto prazo. É por isso que a terapia de substituição da nicotina, ao oferecer uma dosagem apropriada para atender às necessidades de cada organismo, pode minimizar os sintomas da desintoxicação.

Depois, há a dependência psicológica. Neste caso, o cigarro tem efeitos psicoativos resultantes de prazer, relaxamento, estímulo intelectual, ansiolítico e inibidor do apetite. Será, portanto, onde o trabalho do psicólogo pode ajudar o fumante em suas reflexões sobre o prazer, compreensão das emoções e controle da ansiedade, entre outros. Estes aspectos variam de intensidade e dificuldade de um fumante para outro, mas são decisivos para o sucesso do tratamento.

Ocorre ainda, a dependência ambiental e comportamental, já que o tabagismo está associado às diversas circunstâncias que envolvem pessoas e lugares. Desse modo, a mudança de hábitos e rotina podem ajudar no tratamento. O tabagismo está fortemente sugerido nos diferentes ambientes e comportamentos, porque tem um papel social relacionado ao tempo de partilha - de cigarros e de sensação. É importante que o sujeito faça uma lista de lugares ou situações, nos quais ele gosta de fumar para assim antecipar ou evitar as situações em que ele estará mais sensível às recaídas.

O objetivo do tratamento é ajudar a pessoa, em qualquer contexto, a aprender a viver sem tabaco e, para isso, os consumidores de tabaco precisam ser acompanhados por um longo período e nas diferentes fases que envolvem o consumo, entre as quais se destacam: prevenção, uso esporádico, consumo ativo, consumo abusivo, dependência, redução do consumo, abstinência, recaídas e manutenção da cessação do tabagismo(22).

À luz desses pressupostos empíricos e teóricos, o presente estudo buscou identificar variáveis clínicas e razões tabagistas para usuários buscarem tratamento especializado, na luta contra o tabagismo. O propósito de conhecer o perfil dessa população é de, em um segundo momento, ampliar e melhorar os dispositivos de prevenção, intervenção e acompanhamento terapêuticos no cenário da saúde pública do Brasil.

\section{Método}

Trata-se de um estudo retrospectivo documental, de abordagem quantitativa, tendo como fonte todos os prontuários de pacientes dependentes de nicotina em um Centro de Atenção Psicossocial Álcool e outras Drogas-CAPS-AD, localizado em uma cidade, localizada no noroeste do Estado do Rio Grande do Sul, desde a criação, no ano de 2013, até o ano de 2016. 
A coleta dos dados abrangeu variáveis sociodemográficas, história do tabagismo, presença de comorbidades clínicas e sintomas psiquiátricos (diagnosticados pelo médico da unidade), uso de medicação e as razões para busca de tratamento. 0 grau de dependência ao cigarro foi avaliado pela escala Fagerstrom (23), composta de 6 questões relacionadas ao tempo do hábito de fumar, desde o primeiro cigarro do dia, o número de cigarros diários, o cigarro que mais satisfaz e em que período do dia, e frequência do uso. Quanto mais elevado o escore da soma das pontuações de cada resposta dada (que variam de 0 a 3 pontos), maior é o nível de dependência.

Como procedimentos, inicialmente contatou-se o diretor responsável pela instituição, o qual assinou a Carta de Autorização para a realização deste estudo. Na sequência, a pesquisa obteve a aprovação do Comitê de Ética em Pesquisa (CEP) da Universidade de Passo Fundo, sob parecer de número 58594116.7.0000.5342; e em posse destes documentos, realizou-se a análise documental dos protocolos. Os dados foram categorizados de acordo com as variáveis sociodemográficas como gênero, idade, estado civil e escolaridade; e clínicas como histórico de uso de tabaco, presença de comorbidades, motivação para a busca de tratamento. A análise dos resultados contou com a estatística descritiva e a literatura pertinente. As proporções foram descritas com os respectivos intervalos de confiança de $95 \%$, estimados utilizando-se o método exato de Fischer.

\section{Resultados}

Dos 211 usuários que buscaram o serviço desde a sua instalação, no ano de 2013 até 2016, verificou-se que 41 apresentaram dependência relacionada à nicotina. A Tabela 1 apresenta as características sociodemográficas e clínicas dos participantes, bem como as razões para buscar tratamento.

Tabela 1 - Descrição das variáveis sociodemográficas e clínicas dos participantes e razões para iniciar o tratamento $(n=41)$. Cruz Alta, RS, Brasil, 2016

\begin{tabular}{|c|c|c|c|}
\hline Variáveis sociodemográficas & $\mathbf{n}^{*}$ & $\%^{+}$ & IC $95 \%^{*}$ \\
\hline \multicolumn{4}{|l|}{ Sexo } \\
\hline Feminino & 28 & 68,3 & $51,9-81,2 \%$ \\
\hline Masculino & 13 & 31,7 & $18,1-48,1 \%$ \\
\hline Casadas & 22 & 53,7 & $37,4-69,3 \%$ \\
\hline Ensino fundamental Incompleto & 29 & 70,7 & $54,5-83,9 \%$ \\
\hline Atividade remunerada & 26 & 63,4 & $46,9-77,9$ \\
\hline Variáveis clínicas sobre o tabagismo & $\mathrm{n}^{*}$ & $\%^{+}$ & \\
\hline \multicolumn{4}{|l|}{ Início do Hábito de fumar em anos de idade } \\
\hline $10-14$ anos & 14 & 34,1 & $20,0-50,6$ \\
\hline $15-18$ anos & 20 & 48,8 & $32,9-64,9$ \\
\hline $19-36$ anos & 7 & 17,1 & $7,6-32,1$ \\
\hline \multicolumn{4}{|l|}{ Grau de dependênciaş } \\
\hline Baixo & 7 & 17,1 & $7,6-32,1$ \\
\hline Médio & 8 & 19,5 & $8,8-34,9$ \\
\hline Elevado & 12 & 29,3 & $16,1-45,5 \%$ \\
\hline Muito elevado & 13 & 31,7 & $18,1-48,1$ \\
\hline Tentaram parar de fumar de 1 a 3 vezes & 21 & 51,2 & $35,1-67,1$ \\
\hline Ficaram 1 vez sem fumar por pelo menos 1 dia & 17 & 41,5 & $26,3-57,9$ \\
\hline Fumam de 11 a 20 cigarros por dia & 15 & 36,6 & $22,1-53,1$ \\
\hline \multicolumn{4}{|l|}{ Morbidades } \\
\hline Doença clínica atual & 32 & 78,0 & $63,4-89,4$ \\
\hline Sintomas ansiosos atuais & 24 & 58,5 & $0,42-0,74$ \\
\hline Sintomas depressivos atuais & 8 & 19,5 & $8,8-34,9$ \\
\hline \multicolumn{4}{|l|}{ Razões para ter procurado o tratamentoll } \\
\hline Preocupações com a saúde & 35 & 85,4 & $70,8-94,4$ \\
\hline Questões financeiras & 30 & 73,2 & $57,1-85,8$ \\
\hline Pressão familiar & 20 & 48,8 & $57,1-85,8$ \\
\hline Fumar é antissocial & 10 & 24,4 & $12,4-40,3$ \\
\hline
\end{tabular}

$*_{n}$ : frequência absoluta; †\%: frequência relativa; $\neq$ IC 95\%: intervalo de confiança de 95\%; §Avaliado pela Escala Fagerstrom; $\|$ O paciente poderia dar mais do que uma razão 
Verifica-se na tabela 1 que dos 41 pacientes que apresentaram dependência à nicotina, a maior parte são mulheres $(68,3 \%)$, com baixa escolaridade $(70,7 \%)$; início do tabagismo em idades entre 15 e 18 anos $(48,8 \%)$ e 10 e 14 anos (34,1\%), com graus elevados e muito elevados de dependência à nicotina (61\%), altas taxas de comorbidades (78\%) e presença de sintomas de ansiedade (58,5\%). Preocupações com a saúde $(85,4 \%)$ e custos elevados para manter o hábito de fumar $(73,2 \%)$ foram as motivações principais para a busca de tratamento.

\section{Discussão}

Em relação aos dados obtidos neste estudo, quanto ao gênero, diversos estudos ${ }^{(11,16,19,21)}$ que analisaram essa temática chegaram a resultados semelhantes, indicando que as mulheres fumam mais comparadas aos homens. Uma pesquisa(16) relata que na busca de atendimento para a cessação do tabagismo, também há o predomínio de mulheres, indicando que isso se deve, em parte, ao fato de que as mulheres buscam aconselhamento médico com mais frequência e acreditam mais no fato de que o tabagismo cause doenças.

Outra investigação(20) menciona que o gênero feminino, a maior nível de instrução, intenção de parar de fumar e a autoeficácia da cessação tabágica foram positivamente associados à abstinência de fumar, enquanto a dependência da nicotina e a presença de parceiro fumante foram negativamente associados. No mesmo estudo(20), em comparação com a avaliação apenas, o aconselhamento médico foi menos eficaz para pessoas sem intenção de parar de fumar e para desempregados.

No presente estudo, observou-se maior prevalência do gênero feminino e idade média para o início do tratamento de 45 anos para os fumantes que buscaram tratamento para a cessação do tabagismo no CAPS, de forma semelhante ao verificado em outros estudos(16).

Ao considerar o sexo, uma pesquisa(10) revela que entre os indivíduos que abusam de álcool e outras drogas, houve maior prevalência de associação do TMC com as mulheres, indicando que estas apresentaram taxas mais elevadas para TMC, sobretudo os transtornos de humor. Os motivos pelos quais a mulher inicia e se mantém fumando variam conforme a idade, seus fatores psicológicos, socioeconômicos, demográficos e culturais, assim como pela ação da propaganda da indústria do tabaco. Quanto mais precoce a iniciação e maior o tempo de consumo, mais difícil é a cessação(21).

As evidências reforçam a percepção de que a tributação é de fato um instrumento fundamental no controle do consumo de cigarros. No caso específico das mulheres, preços mais elevados ${ }^{(17)}$ tem alta influência, como pode ser observado neste estudo onde $73 \%$ dos participantes indicaram as questões financeiras entre as razões para deixar de fumar.

Outro estudo(18) também conclui que o valor gasto com tabaco causa impactos expressivos no orçamento doméstico, sendo que esses valores poderiam ser direcionados para atender outras necessidades de família. Além disso, conhecer as diversas estratégias de monitoramento, prevenção e combate ao tabagismo, dará um perfil de sua população e pode tornar a estratégia mais eficaz, corroborando com o presente estudo.

É possível que a proporção encontrada na amostra quanto à variável sexo, isto é, mais que o dobro de mulheres em relação ao número de homens -, seja semelhante à da população de pacientes que procuraram o tratamento para a cessação do tabagismo nas unidades públicas de saúde e essa distribuição é compatível com outros achados ${ }^{(21)}$.

Ao considerar os indivíduos com transtornos de ansiedade, um estudo(12) constatou que a maioria era do sexo feminino, tinha entre 26 e 45 anos, segundo grau completo, era casada e estava empregada, vindo ao encontro do verificado no presente estudo.

A literatura ${ }^{(15)}$ descreve que ocorre diferença estatística entre os grupos nas variáveis estado civil, tentativas anteriores de parar de fumar, quantidade de cigarros fumados por dia, uso de fármacos e transtorno do humor referidos. Além disso, o grupo de pacientes abstinentes eram predominantemente composto de casados, com histórico de mais tentativas empreendidas para parar de fumar, com menos cigarro/dia fumados, e apresentaram menos ansiedade/ alteração do humor.

Ao avaliar ${ }^{(24)}$ atitudes, conhecimento e crenças sobre o tabagismo, com uma amostra de 556 acadêmicos de medicina, é relatado alto nível de concordância com medidas efetivas de controle do tabagismo recomendadas pela Organização Mundial de Saúde. Quanto aos métodos de cessação do tabagismo, a maioria dos alunos conhece mais sobre terapia de reposição de nicotina do que sobre terapias não-nicotina ( $93 \%$ vs. $53 \%$ ) e apenas $31 \%$ relataram que acreditam na eficácia de incentivar seus pacientes, durante as visitas médicas.

Retomando o objetivo proposto por este estudo, de identificar variáveis clínicas e razões para busca de tratamento de tabagistas, os resultado obtidos revelam além da predominância do gênero feminino $(68,2 \%)$, a preponderância da baixa escolaridade, o que corrobora achados de estudos prévios ${ }^{(3,14-15)}$.

Identificou-se na literatura relato ${ }^{(14)}$ de que o tempo de uso de tabaco na vida dos pesquisados atingiu um total de 60 anos e, apesar do histórico de tentativas de cessação do tabagismo anterior à participação no tratamento, nenhum participante apresentou menos de 14 anos de uso de tabaco. Apenas 17 pacientes (27\%) declararam que tinha sido a primeira vez que tentavam mudar esse comportamento. 
Em relação às morbidades apresentadas pelos pacientes participantes da presente pesquisa, verifica-se prevalência de doença clínica atual e sintomas ansiosos atuais, como também constatado em diversos estudos $(2,5,8-11,14-15)$.

A literatura(3) refere que, entre as doenças cardíacas, o Infarto Agudo do Miocárdio (IAM) é prevalente entre fumantes e responsável, também, por uma pior qualidade de vida nesse subgrupo. Além disso, a cessação do tabagismo de forma repentina pode expor paciente com IAM a níveis elevados de estresse psicológico, e essa informação é imprescindível para uma avaliação contínua e investigativa relacionada com a gestão de abstinência de nicotina após o IAM, devido ao potencial impacto negativo do estresse psicológico sobre os resultados físicos nesses pacientes ${ }^{(3)}$.

Em uma intervenção ${ }^{(14)}$ com uma amostra de 63 pessoas que tinham participado de um grupo para 0 tratamento do tabagismo, há no mínimo 6 e no máximo 12 meses, em unidade pública de saúde do Distrito Federal, onde foram considerados como critérios de inclusão: ter mais de 18 anos, possuir no mínimo a terceira série do ensino fundamental e ter comparecido a três ou mais encontros do grupo de intervenção, foram identificadas preocupações com a saúde (85\%) entre as razões apresentadas pelos participantes para parar de fumar e buscar tratamento, corroborando vários estudos $^{(2-5,12-13,16,19)}$

Com base em dados prospectivos, a partir de amostra com idosos ${ }^{(4)}$, foram examinadas características de fumantes por raça e sexo, levando a concluir que o comportamento de cessação do tabagismo nesta população é melhor explicitado por maiores níveis de angústia e problemas de saúde, independentemente da raça ou gênero. Esses achados podem ter importantes implicações no tratamento dos programas de cessação do tabagismo entre os idosos. Fumantes idosos com maiores níveis de sofrimento psicológico e problemas de saúde podem estar mais motivados a parar de fumar do que aqueles com menos problemas. Estas dificuldades devem ser orientadas no contexto do protocolo de cessação do tabagismo. Além disso, o mesmo estudo(4) identificou um subgrupo de fumantes idosos que relatam saúde razoavelmente boa, níveis mais baixos de sofrimento e menos propensão a parar de fumar.

O prejuízo à própria saúde e/ou a prevenção está entre os principais motivos que levaram os participantes de um estudo ${ }^{(14)}$ a procurarem ajuda profissional visando mudança do comportamento de fumar. Tais achados são concordantes com os resultados do presente estudo, em que $85 \%$ da amostra indicou, entre as razões de buscar tratamento, as preocupações com a saúde.

É relatado pela literatura(5) que cerca de $50 \%$ dos pacientes psiquiátricos fumam, em contraste com os $25 \%$ da população geral, e que $50 \%$ da população geral consegue parar de fumar, enquanto somente $15 \%$ dos pacientes psiquiátricos o fazem, corroborando com os dados deste estudo.

Ao investigar a associação prospectiva entre tabagismo e depressão, uma pesquisa(10) relata que a maioria são mulheres que fumam mais e que a ansiedade não apresenta consistência, em termos do vínculo da associação mais fortemente apoiada.

Também é apontado pela literatura( ${ }^{(16)}$ que a média de idade para fumantes e não fumantes é de 45 e 44 anos, respectivamente, sendo que as mulheres predominaram nos dois grupos. Os fumantes, com mais frequência, apresentaram incapacidades laborais e domésticas, presença de outros fumantes em casa, hospitalizações, transtorno depressivo, uso de sedativos, história de transtorno mental na família.

Quanto às influências de pais fumantes no desenvolvimento do hábito de fumar de seus filhos, identifica-se na literatura(7) o predomínio de verbalizações dos meninos na categoria má influência, tanto para os adolescentes não fumantes quanto para os adolescentes fumantes. As meninas indicaram a maior frequência na categoria não gosta para as adolescentes não fumantes, e para as fumantes na categoria má influência. Pais tabagistas são alvo de preocupação pela influência negativa que representam para seus filhos, os quais muitas vezes os consideram modelos comportamentais e referências a serem seguidos, e pelos prejuízos à saúde dos familiares que passivamente recebem a fumaça.

A relação entre sintomas de ansiedade, depressão, grau de motivação e cessação do tabagismo é destacada na literatura( ${ }^{(9)}$, por interferir no sucesso do tratamento. Os resultados deste estudo demonstraram que a motivação foi elevada em $50 \%$ dos pacientes, a ansiedade provável em $34 \%$ e $22 \%$ apresentaram depressão provável. A motivação para deixar de fumar foi relacionada com gênero, convivência com fumantes e as tentativas em parar de fumar. A abstinência foi de $59 \%$ na quarta sessão de tratamento e se relacionou significativamente com a idade (pacientes com menos de 30 anos) e com a carga tabágica (consumo menor que 30 maço-ano). A abstinência após seis meses foi de $27 \%$ e se relacionou de forma significativa com menor grau de dependência, menores níveis de ansiedade e depressão e a convivência com fumantes. A motivação elevada influenciou significativamente para a cessação na quarta sessão e após seis meses. Como conclusão o nível de ansiedade e depressão não interferiu na motivação dos pacientes para a busca do tratamento do tabagismo, mas interferiu significativamente na manutenção da abstinência após seis meses do tratamento.

A frequência de transtornos de ansiedade de $75 \%$ foi encontrado na literatura(12), com fobia específica e transtorno de ansiedade generalizada (TAG) sendo os mais frequentes com $26,2 \%$ cada um. Tabagistas 
representaram $21,43 \%$ da amostra, e a maior parte destes obteve escore leve para o grau de dependência, indicando a associação entre ter TAG e ser tabagista, e a chance dos pacientes com TAG fumarem é 5,2 vezes em relação aos que não têm esse transtorno de ansiedade.

Por fim, tabagismo e comorbidades psiquiátricas são problemas humanos complexos que necessitam ser tratados por diferentes correntes do pensamento científico. As diferentes formas de percepção da realidade e outros achados qualitativos ${ }^{(8)}$, devem se somar às informações quantitativas, pois são essenciais na avaliação clínica e no tratamento apropriado, e podem, ainda, reduzir as altas taxas de morbidade e mortalidade associadas ao tabagismo nos pacientes com transtornos psiquiátricos. Assim, os resultados no tratamento desses pacientes podem ser maximizados.

\section{Conclusão}

Diante das características clínicas de dependentes de tabaco evidenciada nesta pesquisa, os resultados deste estudo levam a refletir sobre a necessidade de implementação de políticas para a prevenção e a cessação do tabagismo em mulheres, além de medidas de treinamento dos profissionais de saúde envolvidos no CAPS-AD e de educação da população, desde os escolares. Outras questões a serem consideradas dizem respeito às peculiaridades da mulher em relação ao uso de tabaco e suas vulnerabilidades quanto aos riscos da sua exposição, principalmente no período escolar, gestacional e de amamentação, que devem receber enfoque prioritário.

É relevante ressaltar que os resultados indicam prevalência considerável do consumo de tabaco dos fumantes entrevistados e que a grande maioria anseia por parar de fumar, mas poucos conseguem sem auxílio profissional especializado, o que demonstra a necessidade de oferecer apoio formal para o sucesso dessa busca, assim como inserir ações educativas de prevenção.

Portanto, para cessação do hábito de fumar e o sucesso do tratamento da doença, é essencial a avaliação inicial, além de realizar um programa de intervenção apropriado que deve ser escolhido como auxílio no tratamento, proporcionando programas de prevenção e de tratamento às recaídas. É fundamental o acompanhamento terapêutico, orientando o paciente em todo o processo de cessação do tabagismo.

\section{Referências}

1. World Health Organization. Global status report on noncommunicable diseases 2010. Geneva: World Health Organization; 2011.

2. Wünsch Filho $V$, Mirra $A P$, López RVM, Antunes, LF. Tobacco smoking and cancer in Brazil: evidence and prospects. Rev Bras Epidemiol. 2010;13(2):175-87. DOI: http://dx.doi.org/10.1590/S1415-790X2010000200001. 3. Moreira-Santos TM, Godoy I, Godoy I. Psychological distress related to smoking cessation in patients with acute myocardial infarction. J Bras Pneumol. 2016;42(1):61-7. DOI: http://dx.doi.org/10.1590/ S1806-37562016000000101.

4. Sachs-Ericsson N, Schmidt NB, Zvolensky MJ, Mitchel M, Collins N, Blazer DG. Smoking cessation behavior in older adults by race and gender: The role of health problems and psychological distress. Nicotine Tob Res. 2009;11(4):433-43. DOI: http://dx.doi.org/10.1093/ ntr/ntp002.

5. Nunes SOV, Castro MRP, Castro MSA. Tabagismo, comorbidades e danos à saúde. In: Nunes SOV, Castro MRP. (Orgs.). Tabagismo: abordagem, prevenção e tratamento. $1^{\text {a }}$ ed. Londrina: EDUEL. 2010. p. 224.

6. Cungi C. Faire face aux dépendances: alcool, tabac, drogues, jeux, internet. $1^{a}$ ed. Paris: Retz. 2000.

7. Oliveira CM, Gorayeb R. Diferenças de gênero e fatores motivacionais para início do tabagismo em adolescentes. Saúde Transform Soc. 2012; 3(1):49-54. 8. Calheiros PRV, Oliveira MDS, Andretta I. Comorbidades psiquiátricas no tabagismo. Aletheia. 2006;(23):65-74. 9. Lima MS. Estudo da correlação entre o grau de depressão e ansiedade e a motivação para a cessação do tabagismo [Dissertação]. Brasília: Universidade de Brasília, Mestrado em Ciências Médicas; 2012. 75 f. Disponível em: http://repositorio. unb.br/ handle/10482/10554.

10. Lucchese R, Silva PCD, Denardi TC, Felipe RL, Vera I, Castro PA et al. Common mental disorder among alcohol and drug abusers: a cross-sectional study. Texto Contexto - Enferm. 2017;26(1):e4480015. DOI: http:// dx.doi.org/10.1590/0104-07072017004480015.

11. Fluharty $M$, Taylor $A E$, Grabski $M$, Munafò $M R$. The Association of Cigarette Smoking with Depression and Anxiety: A systematic review. Nicotine Tob Res. 2017;19(1):3-13. DOI: http://dx.doi.org/10.1093/ntr/ ntw140.

12. Munaretti CL, Terra MB. Transtornos de ansiedade: um estudo de prevalência e comorbidade com tabagismo em um ambulatório de psiquiatria. J Bras Psiquiatr. 2007;56(2):108-15. DOI: http://dx.doi.org/10.1590/ S0047-20852007000200006.

13. Lopes FL, Nascimento I, Zin WA, Valença AM, Mezzasalma MA, Figueira I et al. Smoking and psychiatric disorders: a comorbidity survey. Braz J Med Biol Res. 2002;35(8):961-7. DOI: http://dx.doi.org/10.1590/ S0100-879X2002000800013.

14. Martins KC, Seidl EMF. Mudança do comportamento de fumar em participantes de grupos de tabagismo. Psic Teor e Pesq. 2011;27(1):55-64. DOI: http://dx.doi. org/10.1590/S0102-37722011000100008. 
15. Sattler AC, Cade NV. Prevalência da abstinência ao tabaco de pacientes tratados em unidades de saúde e fatores relacionados. Ciênc Saúde Coletiva. 2013;18(1):253-264. DOI: http://dx.doi.org/10.1590/ S1413-81232013000100026.

16. Castro MRP, Matsuo T, Nunes SOV. Clinical characteristics and quality of life of smokers at a referral center for smoking cessation. J Bras Pneumol. 2010;36(1):67-74. DOI: http://dx.doi.org/10.1590/ S1806-37132010000100012.

17. Paes NL. Fatores econômicos e diferenças de gênero na prevalência do tabagismo em adultos. Ciên Saúde Coletiva. 2016;21(1):53-61. DOI: http://dx.doi. org/10.1590/1413-81232015211.00162015.

18. Bazotti A, Finokiet M, Conti IL, França MTA, Vaquil PD. Tabagismo e pobreza no Brasil: uma análise do perfil da população tabagista a partir da POF 2008-2009. Ciên Saúde Coletiva. 2016;21(1):45-52. DOI: http://dx.doi. org/10.1590/1413-81232015211.16802014

19. Schmitz JM, Stotts AL, Mooney ME, Delaune KA, Moeller FG. Bupropion and cognitive-behavioral therapy for smoking cessation in women. Nicotine Tob Res. 2007;9(6):699-709. DOI: http://dx.doi. org/10.1080/14622200701365335.

20. Haug S, Meyer C, Ulbricht S, Schorr G, Rüge J, Rumpf $\mathrm{HJ}$ et al. Predictors and moderators of outcome in different brief interventions for smoking cessation in general medical practice. Patient Educ Couns. 2009;78(1):57-64. DOI: http://dx.doi.org/10.1016/j.pec.2009.07.005.

21. Lombardi EMS, Prado GF, Santos UP, Fernandes FLA. Women and smoking: Risks, impacts, and challenges. J Bras Pneumol. 2011;37(1):118-28. DOI: http://dx.doi. org/10.1590/S1806-37132011000100017.

22. Daver J, Biermé R. Bénéfices À court et à longs termes de la consomation de tabac. Ann Cardiol Angeiol (Paris). 2001;50(4):224-8.

23. Fagerström KO. Measuring degree of physical dependency to tobacco smoking with reference to individualization of treatment. Addictive behaviors. 1978; 3(3-4):235-41. DOI: https://doi. org/10.1016/0306-4603(78)90024-2.

24. Martins SR, Paceli RB, Bussacos MA, Fernandes FLA, Prado GF, Lombardi EMS et al. Effective tobacco control measures: agreement among medical students. J Bras Pneumol. 2017;43(3):202-7. DOI: http://dx.doi. org/10.1590/S1806-37562015000000316.
Recebido: 2.10 .2017

Aceito: 7.12.2018 